\title{
A Hybrid Bacterial Foraging Optimization based on Time-varying Chemotaxis Step and Dynamic Topology Structure
}

\author{
Chen Guo ${ }^{1, a}$, Zhiwen Guo ${ }^{1, b}$, Ben Niu ${ }^{2, c}$, Ying Bi ${ }^{2, d}$ \\ ${ }^{1}$ Business School, Hubei University, Hubei, China \\ ${ }^{2}$ College of Management, Shenzhen University, Shenzhen, China \\ aguochen0714@126.com, bguozhiwen999@126.com, cdrniuben@gmail.com, dwingle1992@qq.co
}

$\mathrm{m}$

Keywords: Bacterial foraging optimization; Time-varying chemotaxis step; Dynamic topology structure.

Abstract. In this paper, we present a hybrid bacterial foraging optimization (HBFO) by combining
the time-varying chemotaxis step strategies with dynamic topology structure. Specifically, we adopt
four kinds of time-varying chemotaxis step methods which use the dynamic topology structure to
improve convergence rate, searching accuracy and optimal value. Four different benchmark functions
are selected as testing functions to compare traditional BFO with HBFOs and the experimental results
validate the efficiency of the proposed algorithms in terms of convergence speed and solution quality.

\section{Introduction}

Bacterial foraging optimization proposed by Passion in 2002 [1] is a swarm intelligent algorithm which inspired by the foraging behavior of Escherichia Coli. In BFO, the bacterial foraging behaviors have three typical models: chemotaxis, reproduction, elimination \& dispersal.

So far, many BFO variants have been developed to improve its performance. Niu et al. [2] proposed two different modified time-varying chemotaxis step methods, i.e., linearly decreasing strategy and non-linearly decreasing strategy to improve the speed of convergence and optimal value. $\mathrm{Gu}$ et al. [3] illustrated an improved BFO by introduced information communicational system in which bacteria share information according to neighbor topologies to slow down the premature convergence. Based on these previous works, a hybrid bacterial foraging optimization algorithm which combines the time-varying chemotaxis step strategies with dynamic topology structure is presented. The rest of the paper is organized as follows. First, the proposed novel BFO algorithms (HBFO) will be described. Next, the performance of proposed BFO algorithms will be tested with four different benchmark functions. Then, we will display the results and analysis. The conclusion will be showed finally.

\section{A Detailed Description of HBFO}

The new time-varying chemotaxis step strategies of improved BFO. In the original BFO, the chemotaxis step length $\mathrm{C}(i)$ is a constant. Hence, it is hard to keep the balance between the local search and global search and affect the searching speed and accuracy. In order to alleviate the drawbacks of traditional BFO, the time-varying chemotaxis step is proposed to make the bacterium have changeable $\mathrm{C}(i)$ in the searching process.

In [2] illustrated two kinds of time-varying chemotaxis step methods, i.e., linearly decreasing strategy and non-linearly decreasing strategy have an excellent performance about the convergence speed and accuracy. As to the non-linearly decreasing strategy, he proposed an exponential decreasing chemotaxis step length strategy (HBFO-E). On this basis, we will present two different non-linearly changing strategies. Finally, the linearly decreasing chemotaxis step length strategy (HBFO-L) and HBFO-E will be compared with traditional BFO together with the two new variants (i.e., HBFO-S, HBFO-N). The variants will be shown as follows. 
The Sine function form of HBFO (HBFO-S). In [4] presented a lot of variants about the inertia weight of PSO, we got the idea from the variants and used the sine function to change chemotaxis step length which is different from the exponential decreasing chemotaxis step length. The step length starts from the minimal value $C_{\text {end }}$ and gradually reaches to the maximal value $C_{\text {start }}$, finally returns to $C_{\text {end }}$. The step length changed in this way to make the bacterium have a local searching ability around them initially and then have a global searching ability by the increasingly stronger cooperation among the bacterium. At last, the bacterium has a local searching ability again. The mathematical formula is presented below.

$$
C(i)=C_{\text {end }}+\left(C_{\text {start }}-C_{\text {end }}\right) * \sin \left(\pi * \frac{j}{N_{C}}\right)
$$

Where $j$ is the current chemotaxis step. $N_{C}$ Is the maximal chemotaxis step.

The normal distribution form of HBFO (HBFO-N). In this novel method, we used the normal distribution changing chemotaxis step length. The step length is a random number that subjects to the normal distribution. The mathematical formula is demonstrated below.

$$
C(i)=\operatorname{normrnd}\left(M U, S I G M A^{2}\right)
$$

Where $M U$ is a mean value and SIGMA is a standard deviation. Meanwhile, the value of $M U$ and SIGMA will be set by the results of the experiments which will be demonstrated in the below.

The dynamic topology structure of improved BFO. In the traditional BFO, there is no information communication among the bacterium. It is easy to slow down the speed of convergence and increase the computational complexity. Many swarm intelligence algorithms, such as particle swarm optimization and genetic algorithm, often use the neighborhood topology structure to communicate with each other to guide the particles to searcher for globally optimal solutions. In [5] proposed a new dynamic topology structure (DTS) which from a full connective topology becoming ring topology once reach to the specified number of iterations. From the results of the experiments, we can know the DTS has an outstanding performance than other static topologies about its convergence speed and optimal results. According to the analysis above, we will combine the DTS with the new time-varying chemotaxis step strategies which are demonstrated above.

A summary of various improved variants of BFO is given in table 1. Meanwhile, the HBFO-N has six kinds of forms (i.e., N1 N6) depended on the value of $M U$.

\begin{tabular}{|c|c|c|c|}
\hline Name & Strategy & Variants & Structure \\
\hline \multirow{4}{*}{ HBFO } & \multirow{4}{*}{$\begin{array}{c}\text { Combine the dynamic } \\
\text { topology structure with } \\
\text { time-varying chemotaxis } \\
\text { step strategy }\end{array}$} & HBFO-L & linearly decreasing chemotaxis step \\
\hline & & HBFO-E & exponential decreasing chemotaxis step \\
\hline & & HBFO-S & Sine function form of chemotaxis step \\
\hline & & HBFO-N & normal distribution form of chemotaxis step \\
\hline
\end{tabular}

Table 1. The Summary of BFO Variants

\section{Experiments and Analysis}

Parameters of benchmark functions. In order to validate the efficiency of the proposed algorithms, four different benchmark functions which include two unimodal functions and two multimodal functions are selected as testing functions to compare original BFO with improved BFO, namely HBFO-L, HBFO-E, HBFO-S, HBFO-N. Table 2 shows the parameter setting about the benchmark functions.

Parameter setting of algorithms. To fairly compare the variants of $\mathrm{BFO}$, we use the same parameters with BFO which are shown in table 3 . The run times of every experiment are 10. Meanwhile, both of the C1and $C 2$ is 2. The $\lambda$ of HBFO-E is 1.7.

Table 2.Global Optimum, Search Ranges And Dimension Of Testing Function

\begin{tabular}{|c|c|c|c|c|c|c|c|}
\hline Fuc & Name & Min Value & Searching Range & Fuc & Name & Min Value & Searching Range \\
\hline$f 1$ & Sphere & 0 & {$[-100,100]$} & $f 3$ & Griewank & 0 & {$[-600,600]$} \\
\hline$f 2$ & Rosenbrock & 0 & {$[-10,10]$} & $f 4$ & Rastrigin & 0 & {$[-5.12,5.12]$} \\
\hline Dim & \multicolumn{7}{|c|}{15} \\
\hline
\end{tabular}


Table 3.The Corresponding Parameter Setting of BFO Variants

\begin{tabular}{|c|c|c|c|c|c|c|}
\hline Variants & S & Nc & Ns & Nre & Ned & Ped \\
\hline N1 N6 & 50 & 100 & 4 & 5 & 2 & 0.25 \\
\hline HBFO & 50 & 1000 & 4 & 5 & 2 & 0.25 \\
\hline
\end{tabular}

Table 4. The Different MU Value

\begin{tabular}{|c|c|c|c|c|c|c|}
\hline Name & N1 & N2 & N3 & N4 & N5 & N6 \\
\hline MU & 0.3 & 0.2 & 0.1 & 0.05 & 0.01 & 0.005 \\
\hline
\end{tabular}

\section{Results and analysis}

The Results and analysis of HBFO-N. As to the equation (2), in order to have a better performance, the parameter $M U$ and SIGMA should be reasonable set. Just as the parameter $C_{\text {start }}$ and $C_{\text {end }}$ is 0.2 and 0.01 , we set the SIGMA is 0.01 and the $M U$ is presented in table 4 .

In order to find the suitable number of $M U$, we use the benchmark functions to test the value in the table 4. After 100 times iterations, we find different $M U$ value suit for different benchmark functions. According to the experimental results, we will choose the $M U$ value which has a best result on benchmark functions to compare with other HBFOs. The summary of $M U$ value on different benchmark functions is given in table 5 .

Table 5. A Summary of MU Value On Different Benchmark Functions

\begin{tabular}{|c|c|c|c|c|c|c|c|}
\hline Fuc & MU & Fuc & MU & Fuc & MU & Fuc & MU \\
\hline$f l$ & 0.05 & $f 2$ & 0.005 & $f 3$ & 0.3 & $f 4$ & 0.05 \\
\hline
\end{tabular}

The Results and analysis of HBFO. Numerical results of HBFO about the mean value and standard deviation of the benchmark functions are listed in table 6. Meanwhile, the mean best fitness value curves for the four algorithms with 10 independent runs for four benchmark functions are plotted in figure 1 to figure 4.

For a more or less comprehensive analyze about the tables and figures, we can come to some conclusions.

1) All of the improved algorithms have a better performance to balance exploration and exploitation for efficiently solving a given optimization problems.

2) As to the function $f 2 f 3$, the HBFO-N demonstrates the superior performance than other improved algorithms about the global optimal value though the convergence speed is slower on function $f 2$.

3) Except for traditional BFO, on function $f 1$, the HBFO-L has an excellent performance about its convergence speed and optimal value. On the contrary, it has a worst performance on function $f 3$. On function $f 4$, the HBFO-E presents a best result and a quick convergence rate than other variants and HBFO-S has a terrible performance.

\section{Conclusion}

In this paper, we presented four hybrid BFO algorithms by combining the time-varying chemotaxis step strategies with dynamic topology structure. On one hand, by using the dynamic topology structure, the bacterium can move toward to global optimal solution by learning the historical best information from itself or others and adjust its position. On the other hand, adding the time-varying chemotaxis step can make the bacterium have a better local and global searching ability during the search process. Otherwise, we used four different benchmark functions to prove the efficiency of the new algorithms compared with the traditional BFO algorithm. By analyzing the experimental results, it is obviously indicated that the improved BFO algorithms have a better performance than traditional BFO.

\section{Acknowledgments}

This work is partially supported by The National Natural Science Foundation of Chi-na (Grants nos. 71001072, 71271140), National Natural Science Foundation of Liaoning province of China (Grant No. 
201102200) and the Shenzhen University Teaching Reform Project (JG2014003, JG2012010).

Table 6 The Results of HBFO on Benchmark Functions

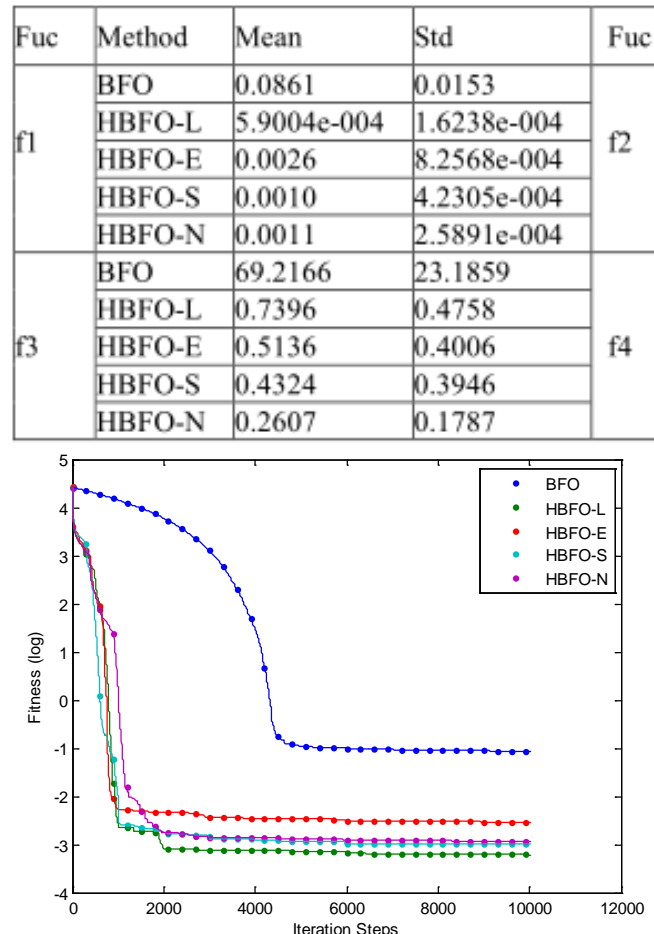

Fig.1.Sphere

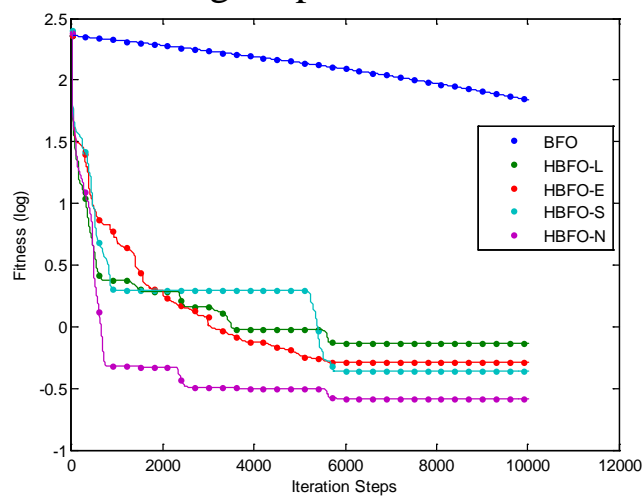

Fig.3.Griewank

\begin{tabular}{|l|l|l|} 
Method & Mean & Std \\
\hline BFO & 19.8154 & 1.9323 \\
\hline HBFO-L & 12.7241 & 1.5752 \\
\hline HBFO-E & 13.6923 & 0.9532 \\
\hline HBFO-S & 12.8154 & 1.7329 \\
\hline HBFO-N & 0.8806 & 1.2847 \\
\hline BFO & 43.2813 & 3.6714 \\
\hline HBFO-L & 20.8376 & 9.2818 \\
\hline HBFO-E & 16.3186 & 4.4668 \\
\hline HBFO-S & 28.8598 & 6.4806 \\
\hline HBFO-N & 20.4134 & 9.6337 \\
\hline
\end{tabular}

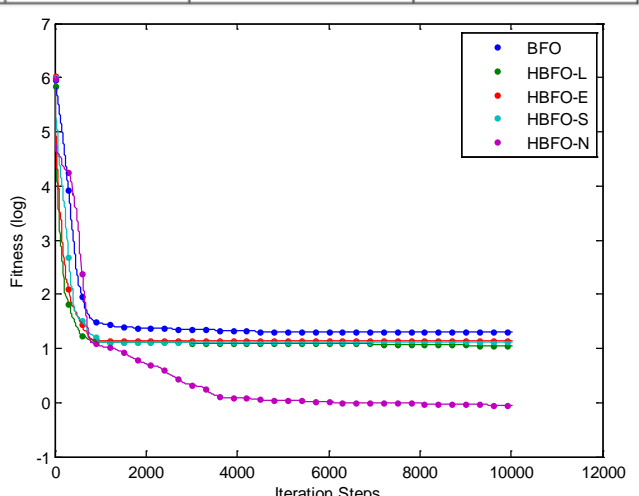

Fig.2.Rosenbrock

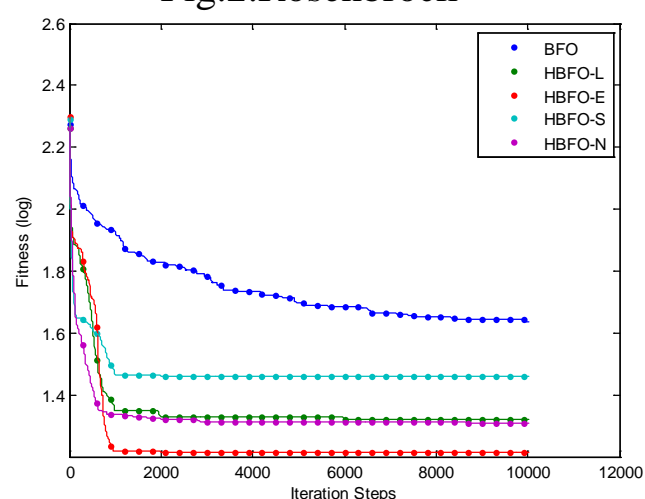

Fig.4.Rastrigin

\section{References}

[1] Passino, K. M.: "Biomimicry of bacterial foraging for distributed optimization and control." Control Systems, IEEE 22.3 (2002): 52-67.

[2] Niu, B., Fan, Y., Wang, H., Li, L., Wang, X.: "Novel bacterial foraging optimization with time-varying chemotaxis step." International Journal of Artificial Intelligence7.A11 (2011): 257-273.

[3] Gu, Q., Yin, K., Niu, B., Xing, K., Tan, L., Li, L.: "BFO with information communicational system based on different topologies structure." Lecture Notes in Artificial Intelligence. 2013. 633-640.

[4] Nickabadi, A., Ebadzadeh, M. M., Safabakhsh, R.: "A novel particle swarm optimization algorithm with adaptive inertia weight."Applied Soft Computing 11.4 (2011): 3658-3670.

[5] Yin, k.: "Studies on the method of network planning optimization faced RFID system." Shenzhen University,2014. 